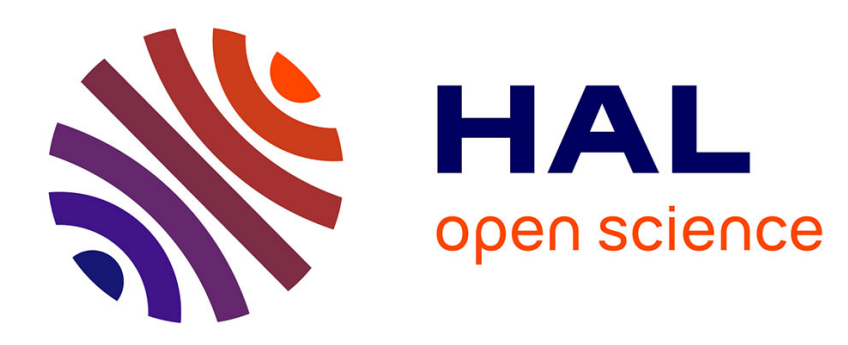

\title{
Effective wall slip in chutes and channels: experiments and discrete element simulations
}

Riccardo Artoni, Andrea Santomaso

\section{To cite this version:}

Riccardo Artoni, Andrea Santomaso. Effective wall slip in chutes and channels: experiments and discrete element simulations. Granular Matter, 2014, 16 (3), pp.377-382. 10.1007/s10035-013-0431y . hal-01470380

\author{
HAL Id: hal-01470380 \\ https://hal.science/hal-01470380
}

Submitted on 17 Feb 2017

HAL is a multi-disciplinary open access archive for the deposit and dissemination of scientific research documents, whether they are published or not. The documents may come from teaching and research institutions in France or abroad, or from public or private research centers.
L'archive ouverte pluridisciplinaire HAL, est destinée au dépôt et à la diffusion de documents scientifiques de niveau recherche, publiés ou non, émanant des établissements d'enseignement et de recherche français ou étrangers, des laboratoires publics ou privés. 


\title{
Effective wall slip in chutes and channels: experiments and discrete element simulations
}

\author{
Riccardo Artoni • Andrea Santomaso
}

Received: date / Accepted: date

\begin{abstract}
Wall slip is an important phenomenon for the flow of granular materials in chutes and channels. The appearance of a slip velocity at the wall critically affects wall stresses and flow profiles, and particularly the total flowrate. In this work we show, through numerical simulations and experiments, that the global slip phenomenon at a wall has peculiar features which deviate significantly from simple sliding behavior. At first we present experimental data for the vertical chute flow which highlight that wall slip depends on many operating and system variables such as flow rate, material properties, wall properties. Secondly, we resume a large campaign of numerical data performed in $2 \mathrm{D}$ with polygonal particles, and try to analyse the effect of material properties, contact parameters, operating variables, different flow configurations, on the slip phenomenon. The numerical campaign allowed to identify the main parameters affecting the wall slip behavior of a numerical model of granular flow, providing the ingredients for the creation of a framework for the description of wall slip.
\end{abstract}

PACS 47.57.Gc $\cdot 45.70 .-\mathrm{n} \cdot 83.80 . \mathrm{Fg}$

\section{R. Artoni}

LUNAM/IFSTTAR

Route de Bouaye CS4 44344 Bouguenais France

Tel.: +33240845616

Fax: +33240845993

E-mail: riccardo.artoni@ifsttar.fr

A. Santomaso

Dipartimento di Ingegneria Industriale

Universita di Padova

via Marzolo 935131 Padova Italy

Tel.: +390498275491

Fax: +390498275470

E-mail: andrea.santomaso@unipd.it
Keywords dense granular flows, wall slip, scaling laws, experiments, discrete numerical simulations

\section{Introduction}

The slip behavior of a granular material densely flowing on a flat frictional boundary is still poorly understood, despite the extensive work done with respect to bulk rheology of dense flows [1] and boundary conditions in other flow regimes [2]. The development of scaling laws for the slip velocity is not only an important theoretical issue which was barely addressed in the past, but also a practical challenge critically affecting the predictions of rheological models. Few works have up to now addressed the problem of interface rheology and wall slip in dense granular systems [3-8].

In this work we present recent experimental and numerical results on wall slip behavior of granular materials, trying to trace a route for the development of such scaling laws. At first, experimental results of vertical chute flow are discussed, for different bulk and wall materials. General features of slip behavior are then analysed, with a particular focus on the joint effect of internal friction, wall roughness and flow rate. In order to understand the underlying contact scale mechanisms, extensive numerical simulations were carried out; in particular, the simulations allowed to study the effect of the internal variables (shear rate, stresses, particle size, ...) on the slip velocity at a wall. Simulations were carried out in 2D for different flow configurations and particle shapes. Relevant dimensionless numbers were identified, and therefore a functional form for the scaling law was proposed, the generality of which was analysed and discussed. 


\section{Vertical chute experiments}

\subsection{Materials and method}

The experimental set-up consisted of a quasi-2D vertical chute, i.e. a rectangular channel (a sketch of which is provided Figure 1a) where one horizontal dimension (which we will call thickness) was much less than the other (the width). The downward motion of the material was controlled by means of a moving plate. An electric motor equipped with a linear reducer was used to control the velocity of the plate. The plate velocity range spanned nearly over two orders of magnitude, from 0.045 to $2.2 \mathrm{~cm} / \mathrm{s}$, in order to test the material behavior in a wide range of flowrates. The channel had a variable width $(10-20-30 \mathrm{~cm})$ and a variable thickness (15-30-45 mm). Data presented here are for the smallest thickness and width; it was verified that the width had no effect on velocity profiles, and thickness was kept small in order to avoid the development of velocity profiles in the transversal direction. Lateral walls (i.e. walls corresponding to the thickness) were roughened with 40 grit and 120 grit sandpaper (corresponding to a grit particle size of $425 \mu \mathrm{m}$ and $125 \mu \mathrm{m}$ respectively). The other two walls were made of glass in order to reduce friction and allow visualization of the flow. A campaign was made with particles of different materials and size, which properties are listed in Table 1 . The values of the angle of internal friction $\phi$ and of the angle of wall friction $\phi_{w}$ were determined using a laboratory shear cell tester, the Brookfield Powder Flow Tester (PFT).

A CCD camera (mvBlueFox) was used for the image acquisition. The camera recorded a zone near a wall, and was driven downward at the same velocity of the moving plate (as depicted in Figure 1a). The frame rate was varied as a function of the plate velocity (from 10 to $25 \mathrm{fps}$ ). At least 300 frames (512x512 pixels) were acquired for each run, representing a square of side $\approx 3 \mathrm{~cm}$. Images were acquired with the vendor software and then processed (cropped, resized, subsequently analyzed) in Matlab. Velocity profiles were computed using a cross correlation Particle Image Velocimetry (PIV) technique, with a code derived from the freely available code Matpiv[9]. For each plate velocity (9 values were chosen), the test was repeated three times. The PIV analysis allowed to reconstruct the velocity profile of the granular material in the direction perpendicular to the wall.

\subsection{Results}

According to the literature[10,11], the velocity profile in vertical chute flow corresponds typically to that sketched

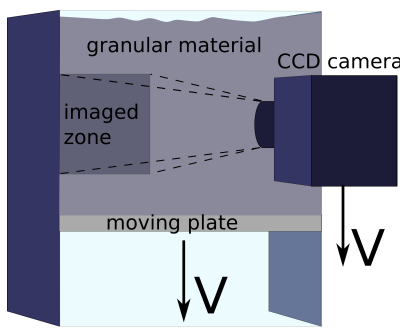

(a)

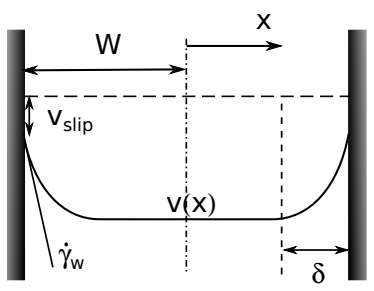

(b)
Fig. 1 (a) Sketch of the experimental setup with the imaging arrangement, (b) Sketch of the typical velocity profile obtained in dense granular flow in vertical chutes.

with its characteristic variables in Figure 1b: shear is localized near the walls, in a shear band which width $\delta$ is of 5 to 10 particle diameters, while the rest of the material is in plug flow. In this work we are concerned with the slip behavior at the wall; a discussion on the full velocity profile will be therefore given elsewhere. For present, we will be content to say that we recovered the typical qualitative velocity profiles referenced above also in our experiments.

In order to characterize wall slip from velocity profiles, it was chosen to use the slip length $\lambda$, a quantity which was suggested already by Navier [12], and which is defined by the ratio between the slip velocity $v_{\text {slip }}$ and the velocity gradient at the wall, $\dot{\gamma}_{w}=d v /\left.d x\right|_{x=W}$ :

$\lambda=\frac{v_{\text {slip }}}{\dot{\gamma}_{w}}$.

In the following we will refer to a dimensionless slip length $\lambda / d_{p}$. From tests performed with the same material and different particle sizes, it was indeed verified that the slip length scaled with particle diameter. This was not surprising, since the particle diameter is the only intrinsic length scale of a granular system. Results from the experiments were sometimes difficult to interpret, because the rescaled slip length appeared to depend generally on many system parameters such as flowrate, internal friction of the material, wall roughness (or wall friction coefficient). An accurate analysis of the results revealed that the materials used for the tests could be divided in two classes determined by the value of the angle of internal friction: class $\mathrm{A}$, characterized by less frictional materials, for $\phi<34^{\circ}$ (iron, steel shots, cellulose), and class B, for $\phi>34^{\circ}$, containing more frictional materials (TAED and calcium carbonate); it has to be noted that the value of $34^{\circ}$ for the transition angle is only given as a crude estimate, due to the discrete nature of the data. Class A materials were characterized by a sharp dependence of the slip length on wall roughness. While for the roughest wall the slip length was found to be independent of flowrate, with values less than 2 particle diameters, for the less 

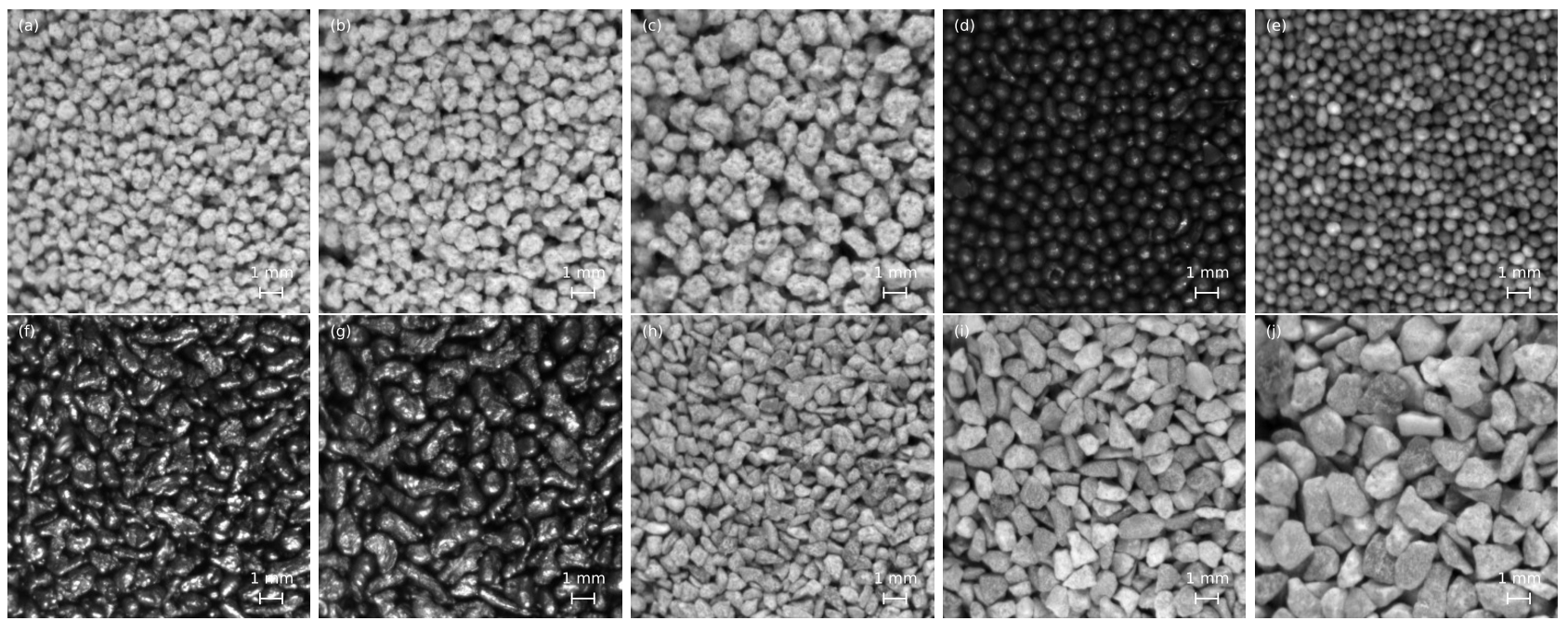

Fig. 2 Photographs of the materials used in the experiments (a) TAED 600-800 (b) TAED 800-1000 (c) TAED 1000-1410 (d) Steel shots (e) Cellulose (f) Steel grit 850-1000 (g) Steel grit 1000-1180 (h) Calcium carbonate 600-710 (i) Calcium carbonate 800-1000 (j) Calcium carbonate 1000-1180.

Table 1 Properties of the materials used for experiments.

\begin{tabular}{|c|c|c|c|c|c|}
\hline Material & sieve size range $(\mu \mathrm{m})$ & $\rho_{b}\left(\mathrm{~kg} / \mathrm{m}^{3}\right)$ & $\phi$ & $\phi_{w}{ }^{\dagger}$ & $\phi_{w}^{\ddagger}$ \\
\hline Calcium carbonate & $600-710$ & 1276 & 36.3 & 36.2 & 34.15 \\
\hline Calcium carbonate & $800-1000$ & 1317 & 37.7 & 36.2 & 31.8 \\
\hline Calcium carbonate & $1000-1180$ & 1281 & 39.5 & 35.8 & 33.2 \\
\hline Granulated cellulose & $600-700$ & 845 & 27.2 & 27.8 & 23.7 \\
\hline Iron (Pometon N60 ) & $710-1000$ & 4319 & 26.5 & 26.2 & 21.5 \\
\hline Steel shots, angular (Pometon Graninox Cr60) & $1000-1180$ & 3834 & 33.8 & 33.8 & 30.6 \\
\hline Steel shots, angular (Pometon Graninox Cr60) & $850-1000$ & 3922 & 33.5 & 33.3 & 31.7 \\
\hline TAED & $600-800$ & 517 & 35.4 & 36.4 & 32.6 \\
\hline TAED & $800-1000$ & 523 & 36.2 & 36.8 & 32.3 \\
\hline TAED & $1000-1410$ & 473 & 37.3 & 36.6 & 32.7 \\
\hline
\end{tabular}

$\dagger 40$ grit sandpaper, ${ }^{\ddagger} 120$ grit sandpaper.

rougher wall the same materials showed a strong monotonic increasing dependence on flowrate, with $\lambda$ starting from around 5 particle diameters up to around 25 particle diameters for the higher velocities. On the other hand, class B materials were always characterized by an increase of the slip length with flowrate, but displayed a weaker dependence on wall roughness. Figure 3 synthetically displays these issues. In order to resume the results, in the figure we plotted the average value of the dimensionless slip length, together with the maximum and minimum values. The relative wall roughness $d_{R} / d_{p}$ is used as a roughness parameter, where $d_{R}$ is the typical dimension of wall roughness elements.

\section{Numerical Simulations}

\subsection{Simulation method and parameters}

The experimental results allowed to appreciate the complex picture associated with wall slip and its main trends.

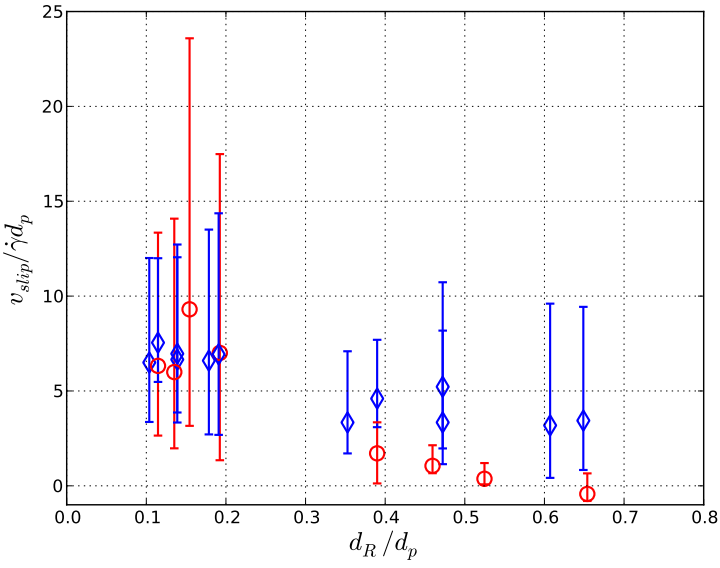

Fig. 3 Dimensionless slip length $\frac{v_{s l i p}}{\dot{\gamma} d_{p}}$ versus relative roughness $d_{R} / d_{p}$, for (०) class $\mathrm{A}$ and $(\diamond)$ class B materials (see explanation in the text). The symbols represent averages of the slip length, while the bars represent the span of the data, from minimum to maximum value. 


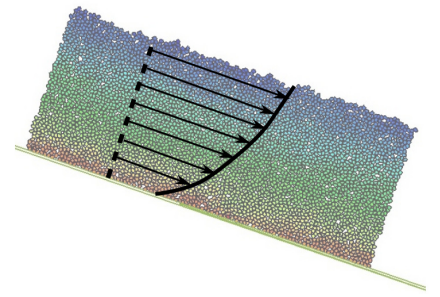

(a)

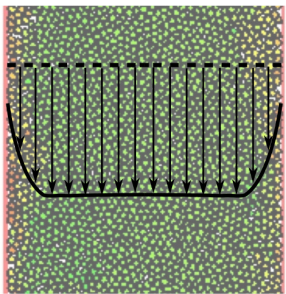

(b)

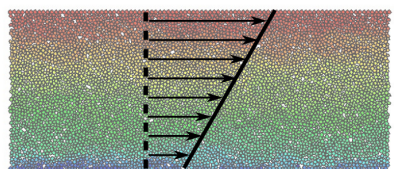

(c)

Fig. 4 Flow configurations used in the numerical simulations: (a) inclined chute, (b) vertical chute, (c) simple shear flow.

In order to refine the analysis and try to determine scaling laws for the slip velocity, discrete numerical simulations were carried out. Focusing on dense granular flows over flat frictional walls, a large plan of 2D simulations was carried out, varying (1) flow configuration, (2) particle-wall friction, (3) particle shape, (4) particle diameter an (5) flow configuration parameters (flow depth and inclination in inclined chute flow, channel width in vertical chute and simple shear flow). We focused on two dimensional simulations, in order to perform a larger number of runs and identify there the scalings to be then tested in 3D. Discrete element simulations were carried out using the Contact Dynamics method developed by Moreau and Jean [13] and implemented in the open source platform LMGC90 [14]. The study was focused on irregular particles (polygons): as a model of polygonal particle with a strong angularity, pentagons were chosen. The initial sample was slightly polydisperse in all the runs, uniformly distributed in a range $d_{p} \pm 0.1 d_{p}$. Interparticle and particle-wall interactions were chosen as completely inelastic, with Coulomb friction for long lasting contacts. While the interparticle friction coefficient $\mu_{p}$ was kept constant and equal to a typical value adopted in literature $\left(\mu_{p}=0.3\right.$, with no connection with the experiments presented above), the particle-wall friction coefficient $\mu_{p w}$ was generally varied, as reported, together with the other simulation parameters, in Table 2. A sketch of the different flow configurations simulated is given in Figure 4. For each test, after the system reached a steady state, continuum averages of velocity and stresses were obtained, as discussed in [8]; subsequent analyses were based on these continuum averaged values.

\subsection{Inclined chute flow}

For the inclined chute flow, the results were discussed in more detail in [8]. If a simple sliding Coulomb boundary condition was valid, the slip velocity should follow the following behavior: for $\tan \theta<\mu_{p w}$ (where $\theta$ is the inclination of the chute) material should experience no-slip at the wall, for $\tan \theta=\mu_{p w}$ a steady sliding phase and for $\tan \theta>\mu_{p w}$ an accelerated sliding phase. In fact, in the simulation results, the average slip velocity was found to be non null for a wide range of $\tan \theta<\mu_{p w}$. In particular, when changing the basal friction coefficient, it was observed that the velocity profile remained unchanged in its shape (with the shear rate having the typical $(H-y)^{3 / 2}$ scaling with depth), but was shifted of a certain slip velocity. This had a strong impact on the inclined chute flowrate, which was generally larger than the one predictable using a simple sliding boundary condition. In order to analyse results, the system variables were made dimensionless. The relevant dimensionless variables at the wall were identified as: (1) the inertial number $I=\frac{\dot{\gamma} d_{p}}{\sqrt{p / \rho_{p}}},(2)$ the particle-wall friction coefficient $\mu_{p w},(3)$ the effective friction coefficient at the wall $\mu_{w}=\tau / \sigma_{w}$ where $\tau$ is the shear stress and $\sigma_{w}$ is the normal stress in the direction perpendicular to the wall, and (4) the dimensionless slip length $\frac{v_{s l i p}}{\dot{\gamma} d_{p}}$.

For the inclined chute configurations (with both regular and elongated particles), a scaling law was found relating three of these four parameters, a good fitting of which was found to be $\frac{v_{s l i p}}{\dot{\gamma} d_{p}}=A\left(\frac{\mu_{w} / \mu_{p w}}{1-\mu_{w} / \mu_{p w}}\right)^{B}$, with best-fit parameters $A=2.2, B=0.42$ for elongated and $A=4.23$ and $B=0.33$ for regular pentagons. The data as well as the fitting functions are shown in Figure 5 .

\subsection{Other flow configurations}

In order to test the validity of the scaling law, inclined chute results were compared with results from other flow configurations: the simple shear and the vertical chute flow. Results are shown in Figure 6. It is first necessary to note that vertical chute simulations, which were steady but not periodic (particles were recirculated at the free surface and velocity was controlled by fixing the velocity of a bottom layer), were locally averaged in space but globally averaged only in time. Therefore each simulation produced a series of values of slip velocity, stresses et cetera, obtained as a time average of the given variable at a certain position at the wall. In order to help comprehension, vertical chute results were therefore represented with error bars in 
Table 2 Parameters defining the numerical simulations.

\begin{tabular}{lccccc}
\hline Flow geometry & Dimension & Particle shape & $\begin{array}{c}\text { Number of } \\
\text { particles } N_{p}\end{array}$ & $\begin{array}{c}\text { Wall Inertial number } \\
I=\frac{\dot{\gamma} d_{p}}{\sqrt{p / \rho_{p}}}\end{array}$ & $\begin{array}{c}\text { Particle-wall friction } \\
\text { coefficient } \mu_{p w}\end{array}$ \\
\hline Inclined chute[8] & 2D & Regular pentagons & 5000 & $2 \times 10^{-2}-10^{-1}$ & $0.63-1.08$ \\
Inclined chute[8] & 2D & Elongated pentagons & 5000 & $5 \times 10^{-3}-1.5 \times 10^{-1}$ & $0.63-1.08$ \\
Vertical chute & 2D & Regular pentagons & $4500-9000$ & $10^{-3}-10^{-1}$ & $0.4-1.2$ \\
Simple shear & 2D & Regular pentagons & 4000 & $10^{-2}-10^{-1}$ & $0.47-1.1$ \\
\hline
\end{tabular}

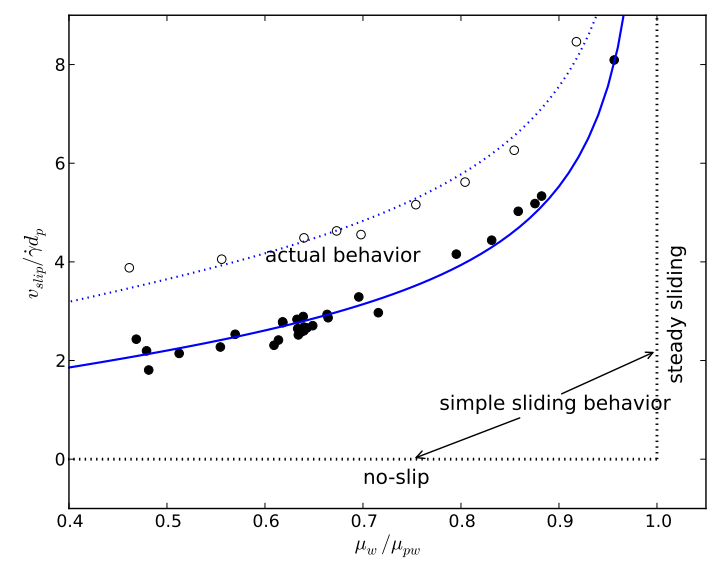

Fig. 5 Dimensionless slip parameter $\frac{v_{s l i p}}{\dot{\gamma} d_{p}}$ versus rescaled effective wall friction coefficient $\frac{\mu_{w}}{\mu_{p w}}$, for (o) regular and (•) elongated pentagons, for inclined chute flow. Lines are best fits described in the text. Reproduced from [8].

figure 6 , while for the other simulations each point corresponds to a simulation. A strong result was found: for high enough friction, $\mu_{w} / \mu_{p w}<0.75$, the scaling law appears to be independent of the geometry. For $\mu_{w} / \mu_{p w}>0.75$, simple shear results agree fairly well with inclined chute results, however strong deviations appear in vertical chute results, characterized also by a larger variance of the results.

\subsection{Scaling universality}

Many instructive issues can be extracted from the numerical results. First, it was found that the scaling law for the slip length preserved the same qualitative features among different geometries. In particular, for all the geometries the behavior is consistent in its limits with simple sliding: the behavior tends to zero slip length for high friction, i.e. $\mu_{w} / \mu_{p w} \rightarrow 0$ ), and to zero shear with a finite velocity for low enough friction. Then, between the two limits ( i.e. $0<\mu_{w} / \mu_{p w}<1$ ), slip velocity and shear rate take generally finite values, with an increasing slip length for increasing $\mu_{w} / \mu_{p w}$. A relation between two dimensionless numbers, $\frac{v_{s l i p}}{\dot{\gamma} d_{p}}$ and $\mu_{w} / \mu_{p w}$, was proposed to describe this intermediate be-

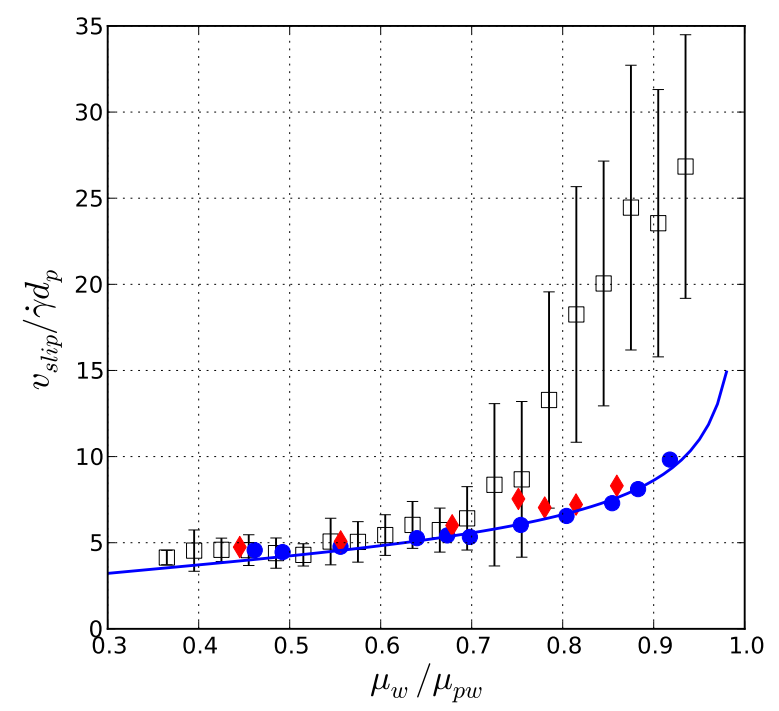

Fig. 6 Dimensionless slip parameter $\frac{v_{s l i p}}{\dot{\gamma} d_{p}}$ versus rescaled effective wall friction coefficient $\frac{\mu_{w}}{\mu_{p w}}$, for regular particles, in different flow configurations: $(\bullet)$ inclined chute flow (Figure $5)$, ( $\square)$ vertical chute flow, and $(\diamond)$ simple shear flow. The solid line is the best fit for inclined chute flow of the scaling law described in the text.

havior. The same functional form was found to hold for different particle shapes, with more irregular particles showing lower slip length values.

For high enough wall friction, the scaling was found to be independent on the geometrical configuration. This suggests that a road for a universal scaling of wall slip has been traced. The deviations observed in vertical chute simulations need to be explained, and this should be probably done by introducing other dimensionless numbers, such as the inertial number, the normal stress ratio, and the solid fraction in the tractation.

\section{Discussion and conclusions}

In this work, at first experiments were presented in order to study the main features of wall slip in dense granular flows. Slip was characterized with a dimensionless parameter, the rescaled slip length $\frac{v_{s l i p}}{\dot{\gamma} d_{p}}$. While a rather complex picture was discovered, the major 
trends were identified: the joint effects of internal friction, wall roughness and flowrate were highlighted. In order to obtain a more refined picture, 2D discrete numerical simulations of polygonal poarticles were carried out in different flow configurations. First, results tend to confirm the theoretical framework firstly proposed in [3]: dense flows of granular materials are characterised by force chains which are continuously breaking and forming. Local, ephemeral force imbalances are therefore to be found in these flows. These imbalances which characterize the dynamics of the force network may cause the tangential forces to be locally higher than the sliding threshold, even if the average tangential force is lower than the average sliding threshold. The kinematics of particles in contact with the wall will be therefore characterised by a succession of slip events, which will globally produce a finite average slip velocity. This framework, applied to the numerical results, allowed to hypothesize that the dimensionless slip length is a function of the ratio between the effective wall friction coefficient $\mu_{w}=\tau / \sigma_{w}$ and the particlewall friction coefficient $\mu_{p w}$. The framework appeared to be consistent among different geometrical configurations, though some deviations were found (particularly for vertical chute results) which strongly need further clarification. Future work will deal with testing of the relevant scaling laws in 3D simulations; in parallel, a plan of experimental tests coupling measurements of velocity profiles and wall stresses is under development.

Acknowledgements RA wishes to thank P. Richard for helpful discussions.

\section{References}

1. G. D. R. Midi, Eur. Phys. J. E 14(4), 341 (2004)

2. M.W. Richman, C.S. Chou, J. Appl. Math. Phys. (ZAMP) 39, 885 (1988)

3. R. Artoni, A. Santomaso, P. Canu, Physical Review E (Statistical, Nonlinear, and Soft Matter Physics) 79(3), 031304 (2009).

4. P. Moucheront, F. Bertrand, G. Koval, L. Tocquer, S. Rodts, J.-N. Roux, A. Corfdir, F. Chevoir, Magnetic Resonance Imaging. 28, 910 - 918 (2010).

5. G. Koval, F. Chevoir,J.-N. Roux, J. Sulem, A. Corfdir, Granular Matter. 13, 525-540 (2011).

6. Z. Shojaaee, L. Brendel, J. Trk, D.E. Wolf, Phys. Rev. E. 86, 011302 (2012).

7. E. Azéma, Y. Descantes, N. Roquet, J.-N. Roux, F. Chevoir, Phys. Rev. E. 86, 031303 (2012).

8. R. Artoni, A. Santomaso, M. Go', P. Canu, Phys. Rev. Lett. 108, 238002 (2012).

9. http://www.math.uio.no/ jks/matpiv/.

10. R.M. Nedderman, C. Laohakul, Powder Technol. 25, 91 (1980)

11. V.V.R. Natarajan, M.L. Hunt, E.D. Taylor, J. Fluid Mech. 304, 1 (1995)
12. C. Navier, Mem. Acad. Roy. Inst. Sci. (1823)

13. M. Jean, Computer Methods in Applied Mechanics and Engineering 177(3-4), 235 (1999).

14. M. Renouf, F. Dubois, P. Alart, Journal of Computational and Applied Mathematics 168(1-2), 375 (2004). 